\title{
Neighborhood Poverty Characteristics of Pertussis Cases among Young Children in San Bernadino County, CA, in 2010-2011
}

\author{
James Napoli $^{1}$, Michele Mouttapa ${ }^{1}$, and Stacey Davis ${ }^{2}$ \\ ${ }^{1}$ California State University, Fullerton, Department of Health Science \\ ${ }^{2}$ County of San Bernardino, Department of Public Health, Infectious Disease \\ Epidemiology
}

\begin{abstract}
Background: The burden of most infectious diseases falls heavily on persons living in neighborhoods of lower socioeconomic status. This study examined the distribution of pertussis among children ages 0-5 years by neighborhood poverty categories and ethnicity in San Bernardino County, California, during an epidemic in 2010-2011. Methods: One hundred twenty-seven cases were identified and their residences were geocoded to determine the poverty rate of the census tract the residence was in. All residences were categorized as belonging to one of four poverty categories: $0-4.9 \%$ of residents (low poverty, reference group), $5-9.9 \%$ (medium low poverty), $10-19.9 \%$ (medium high poverty), and $20-100 \%$ (high poverty). Pertussis incidence rates were calculated for the four categories. Results: Pertussis incidence rates did not significantly vary among the poverty categories. Furthermore the ethnic distribution of pertussis cases did not significantly vary among the poverty categories. Conclusions: The usual socioeconomic disparity found for childhood infectious diseases was not found for pertussis incidence. This may be due to the fact that childhood pertussis vaccinations are state-mandated and publicly-funded, therefore more accessible to children of all neighborhoods. To further reduce pertussis incidence, health education efforts might include the elimination of personal belief exemptions from vaccinations, as was done in California.
\end{abstract}

(c) 2015 Californian Journal of Health Promotion. All rights reserved.

Keywords: pertussis, neighborhood poverty, socioeconomic status, children, geocoding, ethnicity, vaccinations

\section{Introduction}

In general, the burden of most childhood infectious diseases falls heavily on individuals living in neighborhoods with higher poverty levels. For example, influenza hospitalizations are more common among children of higher poverty neighborhoods, and are likely due to the higher infection rates that occur in overcrowded living conditions (Yousey-Hindes \& Hadler, 2011). This study represents the first time, to our knowledge, that U.S. country-wide pertussis incidence data for young children (under five years old) have has been published by neighborhood poverty categories, as determined by census tract information. Among the diseases for which universal childhood vaccination has been recommended, pertussis is the least controlled bacterial vaccine-preventable disease for which infants and young children bear the heaviest consequences (Centers for Disease Control and Prevention, CDC, 2006). Among infected children under the age of one year, almost half are hospitalized. Among the children who are infected with pertussis and are hospitalized, $23 \%$ will get pneumonia, $1.6 \%$ will have convulsions, $67 \%$ will have apnea, $0.4 \%$ will have encephalopathy and $1.6 \%$ will die (CDC, 2015).

Pertussis (whooping cough) is an endemic infectious disease and a cause of childhood morbidity and mortality in the United States, with cycles in disease approximately every three to five years (CDC, 2015). Pertussis is highly infective, with secondary attack rates at $80 \%$ for susceptible household members (CDC, 2012). Transmission of pertussis usually occurs from a susceptible person inhaling aerosolized droplets originating from the respiratory tract of an 
infected person. The incubation period ranges from 4 to 21 days, with an average of 7 to 10 days. Individuals with pertussis are most infectious during the catarrhal phase which includes the first 14 days after onset of cough (CDC, 2012). Untreated infants can remain infective for up to six weeks. With antibiotics, communicability ends after five days of treatment (CDC, 2012). It is believed that pertussis is spread to young children mainly from household adult contacts. More immune adults may interrupt the transmission of pertussis to young children.

\section{Childhood Vaccination}

Childhood vaccination remains the primary strategy to control pertussis and reduce morbidity and mortality. Vaccine efficacy ranges from $80 \%$ to $85 \%$ for pertussis vaccines currently licensed in the United States (CDC, 2012). The mandated California pertussis vaccination school requirement is five doses of DTaP (diphtheria, tetanus and pertussis) before entering kindergarten (Immunization Action Coalition, 2015). In California in 2010, it was estimated that $80 \%$ of children ages $19-35$ months and $93 \%$ of children ages 4-6 years had received at least four doses of the DTaP vaccine (California Department of Public Health (CDPH), 2010; Stokley et al., 2011). This pertussis vaccination compliance rate is similar to the vaccination rates of other childhood infectious disease such as measles, mumps and rubella (CDPH, 2010). One study documented an increase in reported pertussis incidence rates in the six years after receiving the fifth DTaP dose, which suggests that vaccine-induced immunity wanes over time (Tartof et al., 2013). This leaves adolescents and adults susceptible to contracting and transmitting pertussis to younger family members. Also, infants may be too young to be fully vaccinated, or their parents may choose not to vaccinate (CDC, 2006).

\section{Pertussis Epidemic in California}

In 2010, California had the largest number of pertussis cases in 60 years. From 2000 to 2009, the average number of pertussis cases reported per year in the state was 1,117, and the average incidence rate was 3.1 cases per 100,000 state residents. In 2010, there were 9,159 reported pertussis cases, corresponding to an incidence rate of 24.5 cases per 100,000 state residents, which is nearly an eight-fold increase incidence rate compared to the previous nine years (Winter et al., 2012). From 2000-2013 in California, infants younger than six months of age had the highest reported incidence of pertussis and Hispanic infants were overrepresented in this group (Winter et al., 2012). The county of San Bernardino, California was equally affected by this epidemic, as residents of this county also experienced nearly an eight-fold increase in incidence rate (from 0.9 cases per 100,000 residents to 7.1 cases per 100,000 residents; $\mathrm{CDPH}, 2011)$. San Bernardino is one of 58 counties in California and the largest county in the country in land area. In 2010, San Bernardino County had a diverse population of approximately 32\% Non-Hispanic White, 50\% Hispanic, $10 \%$ Black, $7 \%$ Asian and $1 \%$ other race individuals (United States Census Bureau, USCB, 2015).

\section{Socioeconomic Status (SES) Disparities}

Health disparities are usually described by differences in self-reported demographic information such as age, gender, race-ethnicity, place and socio-economic status (SES). Use of race/ethnicity data has been a major means to describe disparities among groups, even though there is little evidence for a differential genetic/ biological basis for the many diseases for which disparities occur (Carlsten \& Burke, 2006).

Traditional SES measures including family income and parent education may be used to explain health status variations among various groups. The association between low SES and illness in childhood is well known. For example, whether assessed by parents' income, education, or occupation, SES is linked to a wide range of health problems, including low birth-weight and infant mortality (Pamuk, et al., 1998). Also, the annual incidence of pediatric influenza-associated hospitalizations in high poverty neighborhoods is at least three times greater than that in low poverty neighborhoods (Yousey-Hindes \& Hadler, 2011). Differences in health behaviors like choosing to vaccinate one's children, involve 
more than just the ability to have health insurance that covers the cost of vaccinations or having a higher income. For example, it has been found that there is no one clear explanation, including differences in SES, of why people make certain health choices, such as the decision to smoke, make healthier food choices, or exercise (Pampel, Krueger \& Denney, 2010). Although the impact of SES-related factors on pertussis is unknown, it was hypothesized for this study that rates of pertussis diagnosis during the 2010 California epidemic varied by SES factors. Specifically we predicted that higher poverty would lead to higher rates of pertussis.

A challenge of using income and wealth measures of SES is a lack of case-level socioeconomic data in most public health passive surveillance systems in the United States, as questions pertaining to SES are not typically asked (Friedman, Hunter \& Parrish, 2002). Currently, all medical providers are required by California law to report pertussis cases to the county public health department when they are suspected or confirmed of having the disease (California Code of Regulations, 2011). Investigations of each case are performed in an effort to reduce the spread of the disease. A potential and relatively inexpensive solution to the problem of absent or limited income and wealthbased socioeconomic data in public health surveillance systems is geocoding cases to neighborhood SES data.

\section{Geocoding and Census Tracts}

Geocoding is the methodology of assigning a census tract code to residential addresses and using neighborhood SES data. The census tract data contains a percentage of people living in poverty according to the poverty definition established by the United States Census Bureau (USCB, 2014). Census tracts are small, relatively permanent statistical subdivisions of a county. They are uniquely numbered with a numeric code and they average about 4,000 inhabitants (USCB, 2014). Census tracts are relatively homogeneous with respect to population characteristics, economic status and living conditions (USCB, 2014). In San Bernardino County, California, there are a total of 369 census tracts (USCB, 2015). Census tracts generally detect socioeconomic gradients more consistently than zip codes (Krieger, Waterman, Chen, Soobader \& Subramanian, 2003; Krieger et al., 2002).

\section{The Present Study}

The purpose of this study was to provide a descriptive epidemiologic analysis of pertussis cases in children under five years old, using census tract-level poverty analysis in San Bernardino County California, during an epidemic in 2010-2011. This age group was chosen because the population data (denominator) for the 0-4 year old age group was readily available from the U.S. Census Bureau, (USCB, 2015) and that infants younger than six months of age had the highest reported incidence of pertussis for this epidemic (Winter et al., 2012).

Our prediction was that the highest incidence rates of pertussis cases would be in neighborhoods of high poverty, consistent with other studies of childhood influenza hospitalizations and cervical cancer precursor rates (Yousey-Hindes \& Hadler, 2011; Niccolai et al., 2013). Additionally, we stratified cases by race/ethnicity, to determine whether each of the four neighborhood poverty categories being studied differed on the racial/ethnic distribution of their cases. We did not examine vaccination status because we did not have this information at the case level; however our assumption was that pertussis incidence may largely be due to lower rates of vaccination.

\section{METHODS}

\section{Surveillance Data Extraction}

The San Bernardino County Department of Public Health conducts ongoing population-based surveillance for laboratory-confirmed pertussis cases among all age groups. We used 2010-2011 data in particular because of the epidemic. The surveillance catchment area for this study was San Bernardino County. Surveillance data were collected from laboratory and physician office reports, as mandated by California state laws and regulations. The public health department then followed up with the parent or legal guardian of each pertussis patient to gather demographic information and recommend control measures. 
Data from the county of San Bernardino public health database was formatted such that each record represented one person or case. The data were obtained under the direction of the infectious disease epidemiologist who was in charge of the database. The data for pertussis cases with an episode date of 2010-2011 were sorted from the master database of all reportable diseases in San Bernardino County. The final data set contained a case identification number, address and race/ethnicity from a total of 134 cases in the under five years old age group.

\section{Geocoding Procedures}

We used the Census Geocoder service from the U.S. Census Bureau to obtain a corresponding census tract for the address of each case. The service is designed for coding a file of addresses to a latitude/longitude coordinate, based upon data that has been loaded into the geocoding engine from a MAF/TIGER benchmark database (USCB, American Fact Finder, AFF, 2014). The 2010 U.S. Census gathered information regarding the percentage of residents, within each census tract in San Bernardino County, that was below the federal poverty level in 2009 (USCB, AFF, 2014). Following the methodology from the Harvard School of Public Health, Health Disparities Geocoding Project (Harvard School of Public Health, 2014), and utilizing 2010 Census data, we categorized each census tract into one of four groups: $0-4.9 \%$ (low poverty, reference group), 5-9.9\% (medium low poverty), 10-19.9\% (medium high poverty), and 20-100\% (high poverty). Based upon the cases' home addresses, we determined which of the four poverty groups each case belonged to. A total of 127 (95\%) of the 134 cases were successfully geocoded by matching an address (to rooftop) to a census tract. There were no cases whose parents/guardians reported post office boxes as their main address.

\section{Incidence Rate Calculations for Each Poverty Category}

Pertussis incidence rates were calculated for each of the four poverty categories. For these calculations, the number of cases in a given poverty category was the numerator. The denominator was the estimated number of children between ages 0-4 years old who lived in a census tract tied to a given poverty category, according to U.S. Census 2010 data for San Bernardino County. The denominator was found by aggregating the total number of children living in each of the census tracts that belonged to the given poverty categories (HSPH, 2014). To obtain a total incidence rate per 100,000 for each poverty category, we divided each numerator by its denominator, and then multiplied this proportion by 100,000 . We then stratified the case by race/ethnicity to determine whether the ethnic distribution of cases varied across the four poverty categories.

\section{Data Analysis}

We followed the data analytic procedures utilized by Krieger et al. and the Public Health Disparities Geocoding Project at the Harvard School of Public Health (HSPH, 2014). We compared incidence rates of the three highest poverty neighborhood category groups to the reference group, which was the lowest poverty group $(<5 \%$ poverty). We computed rate ratios to determine differences between a given neighborhood poverty category with the reference group, as done in other neighborhood poverty investigations using the same methodology (Yousey-Hindes \& Hadler, 2011; Niccolai et al., 2013). Then we used a $Z$ test calculator to compare two population proportions, set for a two-tailed hypothesis and significance level at the .05 level (Social Science Statistics, 2014). This test is used to determine whether two populations (e.g., a low poverty neighborhood compared to a high poverty neighborhood) differed significantly on a single characteristic (pertussis incidence in our study). We inputted the case numbers and population sizes of the given poverty level group and the reference group (the lowest poverty group) to obtain a p-value. A chi-squared test was calculated to determine whether there was a racial/ethnic difference in the distribution of pertussis cases among the four poverty categories (Social Science Statistics, 2014).

\section{Results}

In 2010, the estimated population of San Bernardino County California was 2,035,210, with 158,790 individuals (8\%) in the under five 
year old category. Among those under five years old, 63,169 of them (40\%) lived in one of the 90 census tracts with known cases of pertussis (Table 1). We observed two pertussis seasons, which began in January 2010 and ended in December 2011. Of the 134 cases of laboratoryconfirmed pertussis among children under age five reviewed, a total of 127 (95\%) were successfully geocoded and analyzed (Table 1). Results indicated that incidence rates ranged from 185 per 100,000 in the highest poverty level category to 268 per 100,000 in the lowest poverty category. Although incidence rates appeared to demonstrate a pattern of higher incidence rates in lower poverty neighborhoods, the pattern was not statistically significant. P-values ranged from

\section{Table 1.}

Incidence Rates and Rate Ratios by Poverty Level Category

\begin{tabular}{|c|c|c|c|c|c|}
\hline Poverty Level $_{1}$ & Population $_{2}$ & Cases $_{3}$ & Rate $_{4}$ & Rate Ratio $_{5}$ & $\mathrm{p}_{6}$ \\
\hline Low (0-4.9\%) & 7,828 & 21 & 268 & 1 & Reference \\
\hline Medium Low (5-9.9\%) & 7,774 & 16 & 206 & 0.77 & 0.42 \\
\hline Medium High (10-19.9\%) & 23,230 & 45 & 194 & 0.72 & $0 . .21$ \\
\hline High (>20\%) & 24,337 & 45 & 185 & 0.69 & 0.15 \\
\hline Total & 63,169 & 127 & 201 & 0.75 & \\
\hline \multicolumn{6}{|c|}{$\begin{array}{l}{ }^{1} \text { Percentage of people living in poverty, by census tract, according to federally-defined poverty from the } \\
2010 \text { US Census Bureau. } \\
{ }^{2} 2010 \text { US Census of children under } 5 \text { years old for census tracts in San Bernardino County aggregated } \\
\text { into poverty categories. } \\
{ }^{3} \text { Pertussis cases of under } 5 \text { year olds from the } 2010-2011 \text { pertussis epidemic by census tract aggregated } \\
\text { into poverty categories. } \\
{ }^{4} \text { Cases divided by population times } 100,000 \text {. } \\
{ }^{5} \text { Proportion of each poverty level group divided by the Low (reference) group } \\
\text { Z Test Calculator for } 2 \text { Population Proportions, comparing each poverty level group divided by the } \\
\text { Low (reference) group. }\end{array}$} \\
\hline
\end{tabular}

represented in the medium-low (63\%), medium-

0.15 to 0.42 when each poverty category was compared to the lowest poverty category as a reference group.

Table 2 presents pertussis cases by race/ethnicity and poverty categories. Hispanics comprised $60 \%$ of the cases in this study, while they represent $50 \%$ of San Bernardino County's population. Furthermore Hispanic cases were more high (64\%), and high poverty categories (64\%) compared to the low poverty category (38\%). Conversely, non-Hispanic Whites comprised 23\% of the cases in this study, while they represent $32 \%$ of San Bernardino County's population. Non-Hispanic White cases were more represented in the low poverty category (43\%) compared to the medium low (19\%), medium high (22\%), and Table 2.

Pertussis Cases, by Race/Ethnicity

\begin{tabular}{|c|c|c|c|c|c|c|c|c|c|c|}
\hline \multirow{3}{*}{ Race } & & & \multirow{2}{*}{\multicolumn{2}{|c|}{$\begin{array}{c}\text { Low } \\
(0-4.9 \%)\end{array}$}} & & & & & & \\
\hline & \multicolumn{2}{|c|}{ Total } & & & \multicolumn{2}{|c|}{$\begin{array}{c}\text { Med. Low } \\
(5-9.9 \%)\end{array}$} & \multicolumn{2}{|c|}{$\begin{array}{l}\text { Med. High } \\
(10-19.9 \%)\end{array}$} & \multicolumn{2}{|c|}{$\begin{array}{c}\text { High } \\
(>20 \%)\end{array}$} \\
\hline & $\mathrm{n}$ & $\%$ & $n$ & $\%$ & $\mathrm{n}$ & $\%$ & $\mathrm{n}$ & $\%$ & $\mathrm{n}$ & $\%$ \\
\hline Black & 5 & 4 & 0 & 0 & 1 & 6 & 0 & 0 & 4 & 9 \\
\hline Hispanic & 76 & 60 & 8 & 38 & 10 & 63 & 29 & 64 & 29 & 64 \\
\hline White & 29 & 23 & 9 & 43 & 3 & 19 & 10 & 22 & 7 & 16 \\
\hline Asian & 0 & 0 & 0 & 0 & 0 & 0 & 0 & 0 & 0 & 0 \\
\hline Other & 1 & 1 & 0 & 0 & 0 & 0 & 0 & 0 & 1 & 2 \\
\hline Unknown & 16 & 13 & 4 & 19 & 2 & 13 & 6 & 13 & 4 & 9 \\
\hline Total & 127 & 100 & 21 & 100 & 16 & 100 & 45 & 100 & 45 & 100 \\
\hline
\end{tabular}

1. Percentage of people living in poverty, according to federally-defined poverty from the 2010 US Census data at the census tract level. 
differences in pertussis cases across poverty categories seen here nearly reached statistical significance, with a p-value of 0.07 .

\section{Discussion}

This was the first known study, to our knowledge, that county-wide pertussis data for young children (under five years old) have been examined by neighborhood poverty categories in the United States. While there appeared to be a pattern of children living in low poverty neighborhoods having higher incidence rates of pertussis as compared to children living in high poverty neighborhoods, it was not statistically significant. The findings of this investigation did not concur with other studies that have examined neighborhood level SES characteristics in relation to other outcomes (e.g., childhood influenza hospitalizations, Yousey-Hindes \& Hadler, 2011; and human papillomavirus associated cervical cancer, Niccolai et al., 2013). In both studies, high poverty neighborhoods had greater disease burden.

It is possible that the typical positive correlation between poverty and diseases was not found here because of possible lower vaccination rates in the low poverty neighborhoods. In a study of pediatricians' experience with parental vaccine refusals, suburban physicians caring for families of higher socioeconomic status experienced more vaccine concerns and refusals than less wealthy families (Leib, Liberatos \&Edwards, 2011). It is also possible that vaccination rates have increased significantly in the high poverty neighborhoods, hence reducing the SES-related health disparity that is often observed for other diseases. For example in one study, children of young, minority, higher poverty mothers were more likely to have the full vaccination series recommended by the $\mathrm{CDC}$, including pertussis, for children before entering kindergarten. Furthermore, the mothers were more likely to utilize government-run free clinics and not request personal belief exemptions to waive immunization requirements (Kim, Frimpong, Rivers \& Kronenfeld, 2007).
Our findings justify further investigation of variations in pertussis infection and pertussis vaccination rates according to neighborhood poverty characteristics and ethnicity. Using hypothetical calculations, we determined that if we observed the same incidence rates observed in this study, but had a population size that was twice that of San Bernardino County, the highest poverty category would have had a significantly lower pertussis incidence rate than the lowest poverty category, with a p-value of .04 . When examining individual cases, we found a nearlysignificant interaction between neighborhood poverty and ethnicity on pertussis incidence, such that Hispanic cases tended to occur in higherpoverty neighborhoods, whereas non-Hispanic White cases tended to occur in lower-poverty neighborhoods.

\section{Limitations}

There were some study design limitations that are worth noting. First, we measured neighborhood poverty levels, not individual-level SES, which may have a unique influence on pertussis incidence. Second, our calculations were based upon U.S. Census 2010 data to determine neighborhood poverty rates and population sizes for census tracts. Since neighborhood composition may have changed slightly between 2010 and 2011, there may be some sampling error in the computation of incidence rates during the 20102011 pertussis outbreak. Third, the analyses did not take into consideration who was actually vaccinated. If we had such information, we would have been able to determine whether the disparities in pertussis incidence rates across the different SES categories in this study were associated with lower immunization rates.

\section{Conclusion}

Pertussis is a vaccine preventable disease with a vaccine which is school mandated in California and publicly funded through the federal vaccinefor-children program (Shots for School, 2015, CDC, 2015). The present study suggests that the common disparity found in other diseases for which the vaccines are not mandated and not publicly-funded (Yousey-Hindes \& Hadler, 2011; Niccolai et al., 2013) was not found for childhood 
pertussis. In fact, the non-significant trends in our findings were that higher neighborhood poverty is associated with less pertussis risk. High childhood vaccination compliance rates are especially beneficial in higher poverty neighborhoods, as adult residents in such neighborhoods tend to have lower immunization rates, less access to healthcare, and more crowded living conditions. Such circumstances relate to higher risk of disease communicability, as seen in the other studies (Yousey-Hindes \& Hadler, 2011; Niccolai et al., 2013).
To further reduce pertussis incidence, health education efforts might include the elimination of personal belief exemptions for vaccinations, as was done in California (California Legislative Information, 2015). Further research is needed to determine whether neighborhood poverty characteristics are also related to disease severity (as measured by hospitalization or mortality) and pertussis vaccination rates, and whether these relationships vary by ethnicity. Such research would better inform health education efforts that aim to achieve high vaccination levels in all neighborhoods.

\section{References}

California Code of Regulations (CCR), Reportable Diseases and Conditions, 2011, Title 17, §2500,§2593, §2641.5-2643.20, and §2800-2812. Retrieved from:

https://www.cdph.ca.gov/HealthInfo/Documents/Reportable_Diseases_Conditions.pdf. Accessed Sep 28, 2015.

California Department of Public Health (CDPH). Immunization Levels in Child Care and Schools, Kindergarten, 2010. Retrieved from:

http://www.cdph.ca.gov/programs/immunize/pages/immunizationlevels.aspx. Accessed 1/21/15. Jan. 21, 2015.

California Department of Public Health (CDPH). Pertussis Reports, 2011. Retrieved from: http://www.cdph.ca.gov/programs/immunize/Pages/PertussisSummaryReports.aspx. Accessed Jan. 20, 2015.

California Department of Public Health (CDPH). Reportable Diseases and Conditions, 2014. Retrieved from: http://www.cdph.ca.gov/programs/sss/Documents/Title17Sec2505-01-14.pdf. Accessed Feb. 6, 2015.

California Legislative Information, (2015). SB-277, Public health vaccinations. Retrieved from: https://leginfo.legislature.ca.gov/faces/billNavClient.xhtml?bill_id=201520160SB277. Accessed Nov 6, 2015.

Carlsten, C., \& Burke, W. (2006). Potential for genetics to promote public health: genetics research on smoking suggests caution about expectations. JAMA, 296(20), 2480-2482. Retrieved from: http://jama.jamanetwork.com/article.aspx?articleid=204218. Accessed Feb 22, 2015.

Centers for Disease Control and Prevention (CDC). Epidemiology and prevention of vaccine- preventable diseases, the pink book: Course textbook-12th edition second printing (May, 2012). Retrieved from: http://www.cdc.gov/vaccines/pubs/pinkbook/pert.html. Accessed Jan. 15, 2015.

Centers for Disease Control and Prevention (CDC). Pertussis complications, 2015. Retrieved from: http://www.cdc.gov/pertussis/about/complications.html. Accessed Feb. 6, 2015.

Centers for Disease Control and Prevention (CDC). Preventing tetanus, diphtheria, and pertussis among adolescents: Use of tetanus toxoid, reduced diphtheria toxoid and acellular pertussis vaccines: Recommendations of the Advisory Committee on Immunization Practices (ACIP), 2006. Retrieved from: http://www.cdc.gov/mmwr/preview/mmwrhtml/rr5503a1.htm. Accessed Feb. 6, 2015.

Centers for Disease Control and Prevention (CDC). Vaccines for Children Program (VFC), 2015. Retrieved from: http://www.cdc.gov/vaccines/programs/vfc/index.html. Accessed on Nov 6, 2015.

Friedman, D., Hunter, E., \& Parrish, R. (2002). Shaping a vision of health statistics for the 21st century. Washington, DC: Department of Health and Human Services Data Council, Centers for Disease Control and Prevention, National Center for Health Statistics, and National Committee on Vital and Health Statistics. Available at: http://www. ncvhs. hhs. gov/hsvision/, accessed May, 3, 2006.Retrieved from: http://www.ncvhs.hhs.gov/21st\%20final\%20report.pdf. Accessed Feb. 10, 2015. 
Harvard School of Public Health (HSPH). Public Health Disparities Geocoding Project, 2014. Retrieved from: http://www.hsph.harvard.edu/thegeocodingproject. Accessed Dec. 15, 2014. Immunization Action Coalition. School Vaccination Requirements, 2015. Retrieved from: http://www.immunize.org/laws/\#dtap. Accessed Feb. 6, 2015.

Kim, S., Frimpong, J., Rivers, P., \& Kronenfeld, J. (2007). Effects of maternal and provider characteristics on up-to-date immunization status of children aged 19 to 35 months. American Journal of Public Health, 97(2), 259.Retrieved from: http://www.ncbi.nlm.nih.gov/pmc/articles/PMC1781415/. Accessed Feb. 15, 2015.

Krieger, N., Chen, J., Waterman, P., Soobader, M., Subramanian, S., \& Carson, R. (2002). Geocoding and monitoring of US socioeconomic inequalities in mortality and cancer incidence: Does the choice of area-based measure and geographic level matter? The Public Health Disparities Geocoding Project. American Journal of Epidemiology, 156(5), 471-482. Retrieved from: http://www.ncbi.nlm.nih.gov/pubmed/12196317. Accessed Feb. 10, 2015.

Krieger, N., Waterman, P., Chen, J., Soobader, M., \& Subramanian, S. (2003). Monitoring socioeconomic inequalities in sexually transmitted infections, tuberculosis, and violence: Geocoding and choice of area-based socioeconomic measures--the public health disparities geocoding project (US).Public Health Reports, 118(3), 240.Retrieved from: http://www.ncbi.nlm.nih.gov/pubmed/12766219. Accessed Feb. 16, 2015.

Leib, S., Liberatos, P., \& Edwards, K. (2011). Pediatricians' experience with and response to parental vaccine safety concerns and vaccine refusals: a survey of Connecticut pediatricians. Public Health Reports, 126(Suppl 2), 13.Retrieved from: http://www.ncbi.nlm.nih.gov/pubmed/21812165. Accessed Feb. 1, 2015.

Niccolai, L., Julian, P., Bilinski, A., Mehta, N., Meek, J., Zelterman, D., ... \& Sosa, L. (2013). Geographic poverty and racial/ethnic disparities in cervical cancer precursor rates in Connecticut, 2008-2009. American Journal of Public Health, 103(1), 156-163. Retrieved from: http://www.ncbi.nlm.nih.gov/pubmed/?term=Geographic+poverty+and+racial\%2Fethnic+disparities +in+cervical+cancer+precursor+rates+in+Connecticut\%2C+2008\%E2\%80\%932009. Accessed Feb 9, 2015.

Pampel, F., Krueger, P., \& Denney, J. (2010). Socioeconomic disparities in health behaviors. Annual Review of Sociology, 36, 349. Retrieved from: http://www.ncbi.nlm.nih.gov/pmc/articles/PMC3169799/. Accessed Feb 11, 2015.

Pamuk, E. (Ed.). (1999). Health United States 1998: With Socioeconomic Status and Health Chart Book. DIANE Publishing. Retrieved from: http://www.cdc.gov/nchs/data/hus/hus98.pdf. Accessed Feb. 18, 2015.

Shots for School, (2015). California School Immunization Law, K-12. Retrieved from: http://www.shotsforschool.org/k-12/. Accessed November 6, 2015.

Social Science Statistics. Statistical Calculators, 2014. Retrieved from: http://www.socscistatistics.com/tests/ztest/Default2.aspx. Accessed Dec. 12, 2014.

Stokley, S., Cohn, A., Dorell, C., Hariri, S., Yankey, D., Messonnier, N., \& Wortley, P. (2011).Adolescent vaccination-coverage levels in the United States: 2006- 2009. Pediatrics, peds-2011.Accessed 1/20/15. Retrieved from: http://www.cdc.gov/mmwr/preview/mmwrhtml/mm6033a1.htm. Accessed Feb. 12, 2015.

Tartof, S., Lewis, M., Kenyon, C., White, K., Osborn, A., Liko, J., \& Skoff, T. (2013). Waning immunity to pertussis following 5 doses of DTaP. Pediatrics, 131(4), e1047- e1052. Retrieved from: http://pediatrics.aappublications.org/content/131/4/e1047.short Accesses Feb. 14, 2015.

United States Census Bureau (USCB). American Fact Finder (AFF), 2014. Retrieved from: https://www.census.gov/data/developers/data-sets/Geocoding-services.html Accessed Dec. 15, 2014.

United States Census Bureau (USCB). Geographic products branch: Census tracts, 2014. Retrieved from: http://www.census.gov/geo/education/pdfs/CensusTracts.pdf. Accessed Jan. 22, 2015. 
United States Census Bureau (USCB). State \& county quickfacts, 2015. Retrieved from: http://quickfacts.census.gov/qfd/states/06/06071.html. Accessed Jan. 12, 2015.

Winter, K., Harriman, K., Zipprich, J., Schechter, R., Talarico, J., Watt, J., \& Chavez, G. (2012). California pertussis epidemic, 2010. The Journal of Pediatrics, 161(6), 1091-1096. Retrieved from: http://www.ncbi.nlm.nih.gov/pubmed/22819634. Accessed Feb. 12, 2015.

Yousey-Hindes, K., \& Hadler, J. (2011). Neighborhood socioeconomic status and influenza hospitalizations among children: New Haven County, Connecticut, 2003-2010. American Journal of Public Health, 101(9), 1785. Retrieved from:

http://www.ncbi.nlm.nih.gov/pubmed/?term=Neighborhood+socioeconomic+status+and+ influenza+hospitalizations+among+children\%3A+New+Haven+County\%2C+Connecticu t\%2C+2003\%E2\%80\%932010 . Accessed Feb. 1, 2015.

\section{$\underline{\text { Author Information }}$}

James Napoli, 415 W. Foothill Blvd. \#221 Claremont, CA 91711, phone: 909399 9911, fax: 909399 9933, email: jimnapoli@yahoo.com.

* corresponding author 